\title{
Catching the eye with an abstract
}

\author{
Günter Gauglitz
}

Published online: 16 November 2014

(C) Springer-Verlag Berlin Heidelberg 2014

In the past, libraries were where students and researchers obtained updates on knowledge in science. They spent long hours sitting in libraries: searching, for example, the chemicalabstract service for interesting literature by selected keywords; browsing the printed journals; or looking up the work of competitors to find out what they were currently working on.

Certainly, this was time consuming. However, reading the selected article in the journal normally had other consequences. One was that, as you were browsing the rest of the journal, your attention was drawn by diagrams or headers that invited you to read further or look up further citations, or to learn about an interesting field researched by colleagues. Another was that you started skimming articles in other journals, and thus, attention was always drawn into looking up even more articles and interesting topics. You started with a specific idea and, unexpectedly, came upon other interesting ideas to look up in other journals, and gradually you moved away from the article that you originally intended to read: finding related papers and extending the perspective on your own field of research, even reading papers completely unrelated to the original idea. In this way, you obtained new insights and new ideas that led you to read other articles and, in particular, to apply new ideas and approaches from other fields.

In recent years, this approach has changed.

First, through lack of funds, in most university libraries the number of available journals has shrunk drastically. Only journals read by a larger number of people are available, whereas journals that are too specialized are no longer provided.

Published in the topical collection celebrating ABCs 13th Anniversary.

G. Gauglitz $(\bowtie)$

Institute of Physical and Theoretical Chemistry, Eberhard Karls

Universität, Auf der Morgenstelle 18, 72076 Tübingen, Germany

e-mail: abc.gg@ipc.uni-tuebingen.de
Second, the possibility of searching online databases (e.g., SciFinder or Web of Science) by keywords enables researchers to sit in front of their screens and let the computer assist them in selecting interesting literature, whereby search strategy, implemented keywords, or the contents of the abstract determine access to new information.

Because only a few journals are available in libraries and free access to full articles is only offered in the database for these journals, researchers try to determine from the title and the abstract (when it is available) whether an article is of any interest. In addition, the work of known colleagues is skimmed to stay up to date, and through discussions at congresses and during visits you can obtain additional information on the state of your own field of research. Nevertheless, the possibility of reading articles that were not originally the center of interest is reduced. You therefore have to rely on citations in papers, on eye-catching titles, or on information in abstracts to draw attention to articles that may be of interest. Then copies of these articles can be ordered, for example, through SpringerLink or from other libraries. Another subject that could be discussed here is "Citation Alert", and its consequences in focusing literature searches.

What are the consequences for us when we write an article? Even more than before, we have to use eye-catching titles to draw the attention of potential readers. In addition, the abstract must provide sufficient information to enable readers to decide whether the article is of high or low interest for them. Therefore, publishing increasingly requires ideas on how the title and abstract can draw the attention of potential readers, and it is necessary to provide information that helps the reader to understand the introduction and conclusion of articles. What are the consequences for those who publish and for the journals who want to attract attention to their articles? Those who publish want to draw the attention of all those working in related fields, and even to win new readers for the journal - the higher the impact factor the better (which is also 
in the interest of the authors, because a higher impact factor of the journal results in better standing, at least in our current, hfactor-worshipping world). This means that authors and journals have to take far more care to use expressive titles and abstracts that really attract the attention of the reader. Consequently some typical phrases in titles, including "new method" and "fundamental approach", only use up space. The field of research, the method in short, the compound examined (no long IUPAC definitions!), and a reference to whether the article is theory- or experiment-related are of more interest. The abstract itself does not need to provide details (with the exception of an extraordinary limit of detection), but should enable the reader to quickly understand for which field this article is intended; in which way it is positioned; which method has been used; what are the general results; what are the consequences for the specific field; and, in general, it should even attract the interest of researchers not working in this field, making them read, and cite, the article. Some journals even require graphical "abstracts". Perhaps in the future it will be possible to see these graphics in the abstract when searching SciFinder or Web of Science. However, at present, the written text has to attract the attention of the reader.

Previously, authors were always told to provide as much information as possible in the article. Nowadays, in addition, it

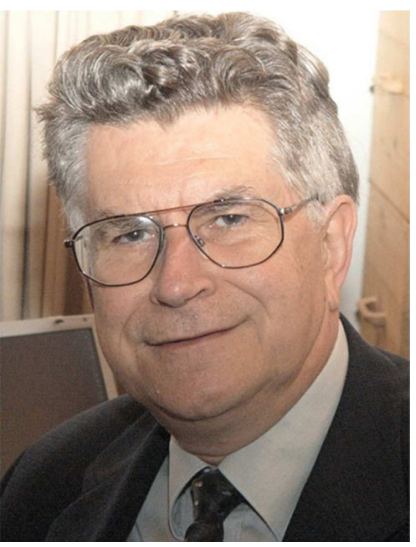

Günter Gauglitz

is Professor emeritus at the Eberhard-Karls-University of Tübingen working in analytical and physical chemistry. He was chairman of the GDCh Division of Analytical Chemistry and chaired the Europt(r)ode VIII meeting. For more than 15 years his main scientific interests have centered on research and development in chemical and biochemical sensors with special focus on the characterization of interfaces of polymers and biomembrane surfaces, spectroscopic techniques, use of spectral interferometry to monitor changes in optical thickness of thin layers, and effects of Fresnel reflectivity at interfaces. He has been Editor of Analytical and Bioanalytical Chemistry (ABC) since 2002.

is also essential to attract the interest of readers without assuming they will be reading the entire article. This means that authors must take more care when composing titles and writing abstracts.

This is my opinion, but I am open to discussing this in more detail in this journal. 